\title{
AVALIAÇÃO DO PROGRAMA DE APOIO À CRIAÇÃO E MODERNIZAÇÃO DE CENTROS VOCACIONAIS TECNOLÓGICOS
}

ASSESSMENT OF THE SUPPORT PROGRAM FOR CREATION AND MODERNIZATION OF VOCATIONAL AND TECHNOLOGICAL CENTERS

\author{
Ivan Rocha Neto
}

\begin{abstract}
Resumo
São apresentados conceitos relativos aos Centros Vocacionais Tecnológicos CVT(s) inseridos nos Programas de Inclusão Social do Ministério da Ciência e Tecnologia (MCT). O artigo visa a discutir os resultados da avaliação do Programa de Apoio à Criação e Modernização dos CVT(s) da Secretaria de Ciência e Tecnologia para Inclusão Social (SECIS), que foi desenvolvida pelo Centro de Gestão e Estudos Estratégicos (CGEE). A metodologia adotada foi qualitativa, com base em estudos de casos, e quantitativa por meio da análise estatística dos resultados dos questionários enviados aos coordenadores dos centros apoiados pela SECIS. Foi feita também a avaliação da inserção dos CVT(s) no Sistema Nacional de Educação Profissional e Tecnológica e são apresentadas considerações em relação às experiências internacionais.
\end{abstract}

Palavras-Chave: Avaliação; Centros Vocacionais Tecnológicos; Experiências Internacionais.

\begin{abstract}
The concepts related to The Vocational and Technological Centers (CVT) in the context of the Social Inclusion Programs of the Ministry of Science and Technology (MCT) are presented. This paper aims to discuss the results of the assessment of the Program for Creation and Modernization of Vocational and Technological Centers of the Secretary for Social Inclusion, which was developed by the Center of Management and Strategic Studies (CGEE). The methodology adopted was qualitative, based on case studies, as well as quantitative by statistical analysis on the results of a questionnaire sent to the Coordinators of the Centers supported by SECIS. A brief assessment with respect to the insertion of these centers in the National System of Professional and Technological Education as well as some considerations on international experiences, are presented.
\end{abstract}

Keywords: Assessment; Vocational and Technological Centers; International Experiences. 


\section{Introdução}

A educação vocacional e tecnológica tem sido foco de políticas públicas em vários países, sobretudo a partir do início do século XIX. No Brasil, as políticas de educação profissional remontam à época do descobrimento e encontram agora inflexão positiva, com a criação dos Institutos Federais de Educação Tecnológica e implantação de novos Centros Vocacionais Tecnológicos - CVT(s) pela Secretaria de Ciência e Tecnologia para Inclusão Social (SECIS) do Ministério da Ciência e Tecnologia (MCT). O processo adquiriu nova dinâmica, em complementação às ações do Ministério da Educação (MEC) e do Serviço Nacional de Aprendizagem Industrial (SENAI).

As atividades de apoio à criação e modernização de CVT(s) estão inseridas no Programa de Popularização e Difusão da Ciência e Tecnologia, que visa a tornar os conhecimentos científicos e tecnológicos acessíveis a uma parcela maior da população. A divulgação científica e tecnológica é uma das dimensões da educação não-formal que desempenha papel importante na formação permanente e na melhoria da qualificação geral científica e tecnológica da sociedade.

Após seis anos de execução do Programa de Criação e Modernização de CVT(s) foi realizada uma avaliação dos resultados do Programa e dos seus processos, considerando que os impactos e sua eficácia não poderiam ser adequadamente avaliados em tão curto espaço de tempo. O objetivo deste artigo é o de apresentar e discutir as conclusões dessa avaliação, que foi desenvolvida pelo CGEE (Centro de Gestão e Estudos Estratégicos) em 2009.

\section{Caracterização e Tipologia dos CVT(s)}

Os CVT(s) são unidades de ensino de ciência, tecnologia e profissionalização, com a finalidade de facilitar o acesso e a difusão e popularização do conhecimento científico e tecnológico. São entidades públicas de caráter comunitário, e têm a missão de educar a população para aproveitamento de oportunidades de inserção profissional e produtiva dos trabalhadores de todas as idades, para pessoas que não têm condições de aproveitar a oferta do sistema de educação formal. A escolha de unidades a serem implantadas considera as realidades e vocações das localidades e regiões onde se inserem, em conexão com diversos outros atores (representantes do governo, trabalhadores, empresas e sociedade), e com os agentes dos Sistemas de Ciência, Tecnologia e Inovação e de Educação Tecnológica, por meio da ação coordenada com as Secretarias Estaduais e/ou Municipais ou com órgãos com missões similares.

Os Centros são caracterizados por condicionantes de enquadramentos no Programa, e envolvem as seguintes finalidades: educação científica, tecnológica e profissional para o mercado de trabalho; e capacitação profissional, voltada ao atendimento de demandas econômicas e sociais das comunidades locais.

Com relação às finalidades e estruturas dominantes, a tipologia adotada no processo de avaliação é brevemente descrita a seguir. 
Tipo A: A finalidade principal é a educação científica e profissional para o trabalho. Destina-se, principalmente às pessoas que não têm condições para aceder ao ensino formal, mas por não terem definidas profissão, ocupação ou capacitação precisam adquirir conhecimentos e práticas para entrada ou permanência no mercado de trabalho.

Tipo B: Capacitação Profissional: a característica dominante é apoiar a capacitação profissional voltada para atender às demandas econômicas e sociais específicas das comunidades locais ou regionais.

Tipo C: Capacitação Profissional e Apoio ao Sistema de Ensino em Ciências.

Há também unidades constituídas para atender simultaneamente a todas essas finalidades, enquanto outras, de estruturas mais simples, atendem-nas parcialmente (tele-centros, estruturas de educação à distância, centros de popularização da ciência e divulgação, informação científica e tecnológica, prestação de serviços tecnológicos, e outros). Todas as unidades estudadas, de forma parcial ou total, enquadram-se nos tipos descritos, mas adotando uma grande diversidade de estruturas.

\section{Inserção no Sistema Nacional de Educação Vocacional e Tecnológica}

O Programa, desde sua criação em 2003, deu sequência ao processo iniciado no Estado do Ceará pelo Deputado Ariosto Holanda (1995-2002). Compreende a implantação de centros com estruturas de comunicação e disseminação de conhecimentos, bem como oficinas e/ou laboratórios destinados a atender às situações particulares de cada localidade.

O sistema de educação profissional e tecnológica nos seus âmbitos político, estratégico e operacional é representado pela figura 1.

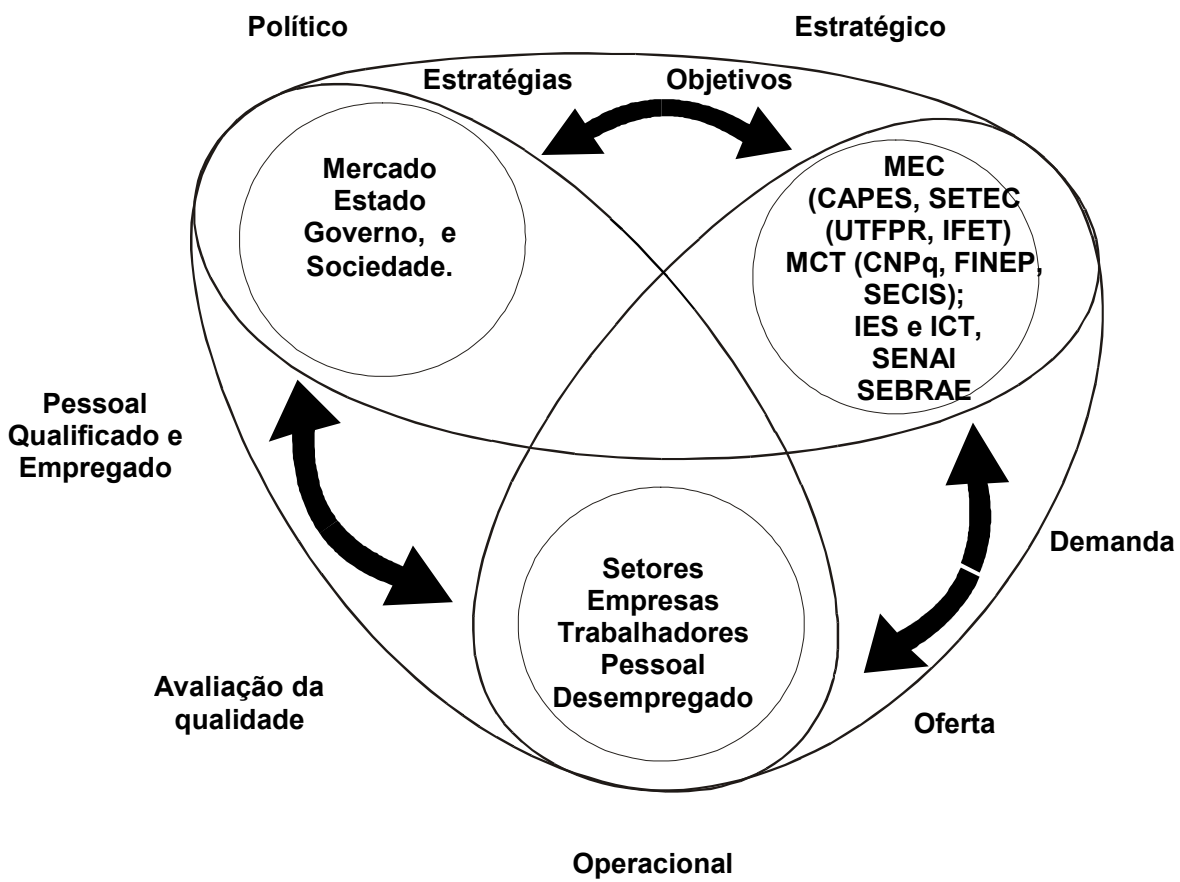


Figura 1. Representação do Sistema de Educação Profissional e Tecnológica Fonte: Rocha Neto (2010)

Há convergências com as políticas de popularização da ciência e da tecnologia, inclusão social e apoio aos arranjos produtivos locais. Nesta avaliação foi observada a complementaridade dos Centros em relação aos cursos oferecidos pelo MEC e pelas unidades de educação da CNI (Confederação Nacional da Indústria), com algumas sobreposições, mas com oferta bem diversificada de vocações e ocupações distintas.

Foi possível identificar a convergência real ou potencial com vários Arranjos Produtivos Locais - APL(s) nos setores de: Alimentos e Bebidas (Carnes, Leite e outros); Automotivo; Construção Civil e Materiais de Construção; Moda e Confecções; Artes e Artesanatos; Agronegócios; Agropecuária e Pesca; Apicultura; Floricultura e Fruticultura; Hotelaria e Hospitalidade; Móveis, Madeiras e Aglomerados; Cafeicultura; Carcinicultura; Couros e Calçados; e, Química (Polímeros, Plásticos, e Cosméticos). Houve ocorrências isoladas de vocações modernas como, por exemplo, Biotecnologia e Mecatrônica. Portanto, também há significativa convergência com os programas de popularização da ciência e tecnologia, inclusão digital e apoio aos arranjos produtivos locais.

Com relação à oferta de cursos do MEC há distinções de níveis e ofertas vocacionais, bem como complementaridades e convergências. Há superposições com os seguintes setores: Construção Civil; Madeira e Mobiliário; Automotivo; Couros e Calçados; Turismo; Metal-Mecânica; e Eletroeletrônica.

\section{Procedimentos Metodológicos}

Além de revisão da literatura sobre educação tecnológica e profissional que deu suporte conceitual para a avaliação do Programa, foi realizado o seguinte percurso metodológico: análise documental; montagem de banco de dados dos projetos de CVT financiados pelo SECIS/MCT; realização de entrevistas com atores envolvidos no processo de implantação do programa e dos projetos; desenvolvimento de indicadores para avaliação; desenvolvimento e aplicação de questionário eletrônico para coleta de informações primárias junto aos representantes dos CVTs e, aferição dos indicadores; tabulações e análises estatísticas do banco de dados, gerado pela aplicação do questionário eletrônico; realização de oficinas de trabalho para construção e validação dos indicadores e dos questionários. Portanto, a metodologia adotada combinou a avaliação quantitativa a partir da análise das estatísticas obtidas da consulta aos coordenadores de centros, com a qualitativa, com base nas informações colhidas nas visitas in loco e entrevistas para fundamentar estudos de casos. Além disso, a metodologia de avaliação se desenvolveu segundo a abordagem sistêmica, discutindo os processos nos âmbitos político, estratégico e operacional (Figura 1), explorando as relações de interdependência e as influências mútuas entre atores e variáveis. Também foram investigadas as experiências de mais de dez países. O objetivo das visitas e das entrevistas foi identificar boas práticas de implantação e operação dos centros bem sucedidos, além de avaliar as condições de funcionamento das unidades implantadas. 


\section{Estudos de Casos}

Os casos estudados foram os seguintes: a Rede CENTECs (Centros de Ensino Tecnológico do Ceará): Centro de Formação de Instrutores (CFI) e Docas, em Fortaleza; e nos municípios de Aracati (CVTEC); Itaiçaba; e, Russas, os dois últimos dedicados à inserção da ciência no trabalho; a Rede Secretaria de Ciência e Tecnologia e Educação Superior do Estado de Minas Gerais - (SECTES) nos seguintes municípios: Ribeirão das Neves - CVT - Henfil mais orientado para educação ambiental; Brumadinho (Polivalente); e, Nova Lima (Educação Ambiental e Hotelaria); a Rede Faetec (Fundação de Apoio à Escola Técnica do Rio de Janeiro); Quintino (Automotivo); Correios (Serviços Gerais); Olavo Bilac (Modas e Confecções); Belford Roxo (Serviços de Manutenção Elétrica e Hidráulica); São João do Meriti (Construção Civil); Nova Iguaçu (Cosméticos); e Mesquita (Construção Civil).

Além disso, foram entrevistados os coordenadores do Ceflora de Cruzeiro do Sul no Acre, em funcionamento, e do Parque Tecnológico da Paraíba, em Campina Grande, cujo projeto de rede ainda será iniciado.

Das visitas e das entrevistas foi possível verificar que as condições de funcionamento estão adequadas na grande maioria dos casos de sucesso investigados e que há boas perspectivas na implantação nos demais.

As seguintes boas práticas foram comuns às unidades implantadas: coordenação integrada às instituições de ensino tecnológico, com vinculação às Secretarias Estaduais de Ciência e Tecnologia e comprometimento político das autoridades estaduais e locais; coerência com as vocações econômicas locais e atendimento às demandas sociais das comunidades atendidas; autonomia pedagógica das unidades; motivação dos coordenadores gerais e dos gestores administrativos, técnicos e pedagógicos (organização padrão); consciência do processo de inclusão social pela educação e trabalho; infraestrutura moderna com projetos padronizados de engenharia e arquitetura; percepção generalizada da sustentabilidade, considerando o apelo político, engajamento das comunidades, e os sucessos obtidos com relação à inclusão social; mesmo face à possibilidade de mudanças dos executivos locais: aprendizagem gerencial com redução do tempo de instalação e funcionamento de novas unidades; existência de projetos pedagógicos supervisionados por profissionais qualificados; disposição de instrutores especializados para atendimento ao público envolvido nas unidades de inclusão digital, existentes em todas as unidades visitadas; e, apoio em diversas formas de parceria, sobretudo em relação ao compartilhamento de pessoal e infraestrutura.

A participação em editais públicos de fomento também foi um indicador promissor de tendência de integração com outros programas e quanto à sustentabilidade das unidades implantadas.

\section{Resultados da Consulta}

Foram consultados 221 coordenadores, tendo-se obtido 86 respostas $(38,9 \%)$. Destes, 59 estavam implantados, o que representa cerca de $50 \%$ do total de unidades em funcionamento no momento da avaliação. Os 
questionários foram organizados para investigar os resultados por dimensões e indicadores de avaliação, conforme descrito a seguir.

\section{Dimensões}

As dimensões de avaliação foram as seguintes: Tramitação do Processo no MCT; Implantação; Operação e Funcionamento; Difusão de Conhecimentos; Infraestrutura; Ensino; Sustentabilidade e Parcerias; e, Interação Sistêmica.

\section{Indicadores}

Para cada uma das dimensões foram escolhidos indicadores cujos resultados são apresentados no quadro 1, que são apresentados sob a forma de percentuais não tendo sido feitas correlações ou medidas estatísticas mais sofisticadas.

Quadro 1: Síntese dos Resultados da Consulta

Dimensão 1. Tramitação dos Processos no MCT Indicador: Relacionamento dos proponentes com o MCT

Os documentos oficiais de orientação para elaboração de projetos estão suficientemente claros e os formulários estão adequados para $95 \%$ O tempo de tramitação de 6 meses a 1 ano foi considerado razoável para 94\%; o tempo médio de implantação inferior a 1 ano e $53 \%$ iniciaram o funcionamento antes da prestação de contas

\section{Dimensão 2. Gênese}

Indicador: Origem dos Projetos

Predomínio da demanda das comunidades locais e também por iniciativa dos arranjos produtivos locais

\section{Dimensão 3. Condições Operacionais}

Indicador: Disponibilidade de docentes

Baixa para as ciências; alta para técnicos de nível médio e superior e informática; baixa para bibliotecários

\section{Dimensão 4. Tipos de Ensino e Público Alvo}

Indicador 1: Oferta

Diversificada, mas predominância de educação de adultos (EJA):

Educação científica; treinamento vocacional; processo seletivo democrático (sorteio ou ordem de chegada)

Indicador 2: Demanda

Mais de $70 \%$ provenientes das comunidades $27 \%$ de APL (s) e empresas.

Indicador 3: População Atendida

Média superior a 1000 estudantes por ano por CVT

Dimensão 5. Sustentabilidade e Parcerias

Indicador 1. Sustentabilidade

Dificuldades de recursos de custeio e manutenção.

Indicador 2. Parcerias

Praticamente $100 \%$ declararam parcerias ou com CEFETs/IFEs ou com 

Instituições.

Dimensão 6. Atividades de Planejamento e Coordenação

Indicador 1. Existência de Projetos Pedagógicos

Apenas $20 \%$ declararam ter elaborado projetos pedagógicos

Indicador 2. Processo de encaminhamento de ex-alunos ao mercado de trabalho

Apenas $10 \%$ desenvolvem processos de encaminhamento dos egressos ao mercado de trabalho

\section{Dimensão 7. Facilidades e Dificuldades de Implantação e Operação}

Indicador 1. Pontos Fortes

Comprometimento dos patrocinadores (21\%); apoio sob a forma de convênio (15\%); interesse dos parceiros (11\%); e, disponibilidade em tempo hábil de instalações e recursos financeiros (19\%)

Indicador 2. Fatores facilitadores

Instalações físicas (17\%); comprometimento dos mantenedores (17\%); elevada demanda dos serviços (11\%); instalação de equipamentos $(10 \%)$ e capacidade e disponibilidade de recursos para pagamento de pessoal $(9 \%)$ Indicador 3. Pontos Fracos

Indisponibilidade em tempo hábil de recursos financeiros e instalações físicas, que somaram $25 \%$ em oposição às facilidades apontadas no item anterior; indisponibilidade de pessoal qualificado para implantação (13\%); dificuldades com os processos de licitação de obras e serviços (12\%); e, dificuldades na execução de obras de reforma e construção (18\%) Indicador 4. Fatores Complicadores

Manutenção de equipamentos (19\%); disponibilidade de pessoal para docência e técnicos (14\%), o que confirma os resultados do item 3; burocracia do serviço público (13\%); e, disponibilidade de recursos para custeio $(13 \%)$

\section{Indicador 5. Infraestrutura}

Praticamente $2 / 3$ informaram que os itens de infraestrutura previstos nos projetos estão concluídos, e que $20 \%$ estão em andamento dentro dos prazos.

Indicador 6. Fluxo Financeiro

$2 / 3$ informaram que há regularidade no fluxo de recursos financeiros

Dificuldades de custeio com despesas maiores que as receitas

Não há autonomia financeira

Fonte: CGEE (2009). Elaboração própria.

Os seguintes aspectos podem ser destacados da consulta: os coordenadores dos centros são em geral adequadamente qualificados, sendo $50 \%$ com graduação, $20 \%$ especialistas, $14 \%$ com mestrado, $12 \%$ com doutorado e apenas $4 \%$, sem nível superior; nos casos implantados predominou a gênese por demanda social $23,7 \%$, seguida da conexão com programas estaduais $(10,2 \%)$ e iniciativas de IES, Cefet, e IFs $(11,9 \%)$; que há forte tendência de interação sistêmica e formação de parcerias (foram identificadas 153 dos 59 centros implantados); a maior demanda que ocorre 
é de treinamento profissional, seguida de atendimento ao público e prestação de serviços de informação; a integração dos CVTs com as unidades da vertente do MEC tem ocorrido sob a forma de parcerias diversificadas. Houve importante melhoria da infraestrutura para educação profissional e tecnológica nos municípios apoiados.

\section{Experiências Internacionais}

Para os objetivos deste artigo, cabe apenas registrar que do estudo das experiências internacionais, cujos resultados foram objeto de outra publicação, o modelo que mais se destacou foi o Dual, concebido e adotado na Alemanha, que integra formalmente a educação profissional e tecnológica com os ambientes das empresas. Esse modelo tem sido adotado mais recentemente em outros países como, por exemplo, China, Países Nórdicos, Reino Unido e França, que mudou o seu sistema, cuja educação vocacional era antes baseada nos Museus de Artes e Ofícios, que inspiraram a experiência brasileira dos Liceus de Artes e Ofícios, e os atuais CVTs. Na China o processo foi muito turbulento durante a Revolução Cultural, mas com a abertura econômica, esse modelo acabou também sendo adotado.

Os estudantes desenvolvem aprendizagem na maior parte do tempo nas próprias empresas e minoritariamente nas escolas técnicas ou universidades (apenas um ou dois dias). Os ambientes de aprendizagem, nos centros vocacionais e nas empresas interagem com ênfases distintas, mas sem divisão nítida ou rígida de funções. Os centros não ensinam somente teoria, mas também refletem sobre as práticas de trabalho. Por outro lado, a aprendizagem nas empresas não envolve apenas a aplicação empírica dos conhecimentos adquiridos. A combinação de aprendizagem no trabalho (teoria e prática) é o fundamento do modelo Dual de educação vocacional e tecnológica.

$\mathrm{Na}$ origem, a formação vocacional, tanto no Brasil como nos EUA, França, Reino Unido e Portugal, ao contrário dos modelos da Alemanha e da Finlândia, teve como motivação comum a inserção no mercado de trabalho de pessoas, física ou socialmente carentes (escravos, veteranos de guerra, jovens em situações de risco, moradores de rua, entre outros). Era, sobretudo, voltada para adultos que não tinham mais condições de aproveitar a oferta dos sistemas formais de ensino profissional.

O processo de descentralização da gestão que ocorreu na Itália se assemelha ao sistema brasileiro de CVTs que articula as esferas federal, estaduais e municipais. Em outros países, notadamente na Alemanha, o processo é coordenado por Comissões Nacionais, envolvendo vários ministérios, representantes dos trabalhadores e de empresários. Adotar esta prática no Brasil poderia ser muito interessante para obter maior sinergia do sistema nacional de educação vocacional e tecnológica e das iniciativas de vários ministérios.

No Brasil, a formação profissional e tecnológica para as empresas tem sido feita basicamente nos cursos oferecidos pelo Senai, e mais recentemente pela instalação de Universidades Corporativas, que não 
necessariamente envolvem a formação de parcerias ou cooperação com as instituições educativas.

\section{Considerações Finais}

No âmbito político, o Programa de apoio aos CVTs tem merecido destacado reconhecimento e prestígio junto ao Congresso Nacional e também por parte do Executivo. O mesmo tem ocorrido nas esferas estaduais e municipais. Há envolvimento das lideranças políticas locais, mediante proposição de emendas parlamentares preferencialmente atendendo às demandas sociais das comunidades beneficiadas. Entretanto, conforme mostrado, o sucesso destes empreendimentos requer forte conexão com as Secretarias Estaduais de Educação, Ciência e Tecnologia e com unidades de educação tecnológica (Universidades, Cefets, Institutos Federais - IFs), de modo a elevar o grau de sustentabilidade das unidades implantadas. Os casos do Ceará, Rio de Janeiro e Minas Gerais são emblemáticos em relação a este quesito. Escolhas têm sido feitas de acordo com as demandas sociais e vocações econômicas locais dos municípios. Foram atendidos mais de 200 nesses seis anos, até o momento da avaliação, confirmando o apelo político suprapartidário e certamente eleva o potencial de sustentabilidade dos centros e do Programa.

Do ponto de vista estratégico, a melhor prática tem sido a formação de redes, com coordenação única, preferencialmente da Secretaria Estadual de Ciência e Tecnologia, e suporte de uma instituição de educação tecnológica, além da necessidade de forte conexão com outros parceiros locais, incluindo empresas. A escolha do coordenador e dos gestores tem sido crucial para o sucesso das redes e não deve ser feita somente por critérios políticos partidários, mas pela competência de gestão e conhecimentos técnicos e educacionais. Unidades isoladas e dependentes do empenho exclusivo dos prefeitos sugerem baixo potencial de sustentabilidade. No âmbito operacional uma relação de boas práticas pôde ser proposta, levando às conclusões a seguir.

Majoritariamente os CVTs cumprem com suas finalidades prestando atendimentos segundo as necessidades das comunidades e vocações econômicas locais.

A principal conclusão que se pode extrair deste processo de avaliação é de que há suficientes evidências para afirmar que o Programa tem sido realizado de forma eficiente, apresentando resultados positivos do Programa para inclusão social e preparação para o mercado de trabalho para a população mais carente.

\section{Agradecimentos}

$\mathrm{O}$ autor agradece ao CGEE pela oportunidade de ter participado como consultor deste Projeto.

\section{Referências}

CGEE. Cadernos de Estudos Técnicos. Avaliação do Programa de Criação e Modernização de Centros Vocacionais Tecnológicos. Brasília: CGEE, 2010. 
SECIS (2009). Secretaria de Ciência e Tecnologia para Inclusão Social. Disponível em: <www.mct.gov.br>.

ROCHA NETO, Ivan. Oferta e Demanda de Pessoal Qualificado no Brasil. In: Formação de Recursos Humanos em áreas estratégicas de Ciência Tecnologia e Inovação. Brasília, DF: Centro de Gestão e Estudos Estratégicos, 2010. 